\title{
Implementação em VHDL de um Modem OFDM
}

\author{
Euripedes Rocha e Jair A. L. Silva
}

\begin{abstract}
Resumo-Neste artigo é apresentada uma implementação, em VHDL, de um modem OFDM aplicado à comunicação via rede elétrica $(P L C)$. Partindo da taxa de bits desejada e do número de sub-portadoras, foi desenvolvido um modem, usando mapeamento 4-QAM, com arquitetura de hardware modular, permitindo uma fácil reconfiguração dos blocos desenvolvidos. Os resultados mostram a taxa de ocupação de harware em função do número de pontos da IFFT/FFT e taxa máxima de clock.
\end{abstract}

Palavras-Chave-OFDM, FPGA, VHDL, PLC.

Abstract-In this paper we present the VHDL implementation of an OFDM modem suitable for Powerline Communications $(P L C)$. Starting from the desired bit rate and number of subcarriers we have developed a 4-QAM modem in a modular architecture, allowing an easy reconfiguration of the functional blocks. The results show the occupation rate of the hardware as a function of the IFFT/FFT size and the maximum clock rate.

Keywords-OFDM, FPGA, VHDL, PLC.

\section{INTRODUÇÃO}

A técnica de multiplexação por divisão de frequiências ortogonais, OFDM (Orthogonal Frequency Division Multiplexing), transformou-se no esquema de transmissão a altas taxas para diversos sistemas de comunicação, tais como acesso à internet em banda larga com e sem fio, comunicação entre periféricos de computadores pessoais, televisão digital e sistemas PLC (Power Line Communications)- comunicação pela rede elétrica. Vários padrões de comunicação definiram em sua camada física a OFDM como técnica de modulação ou codificação de dados [1], [2]. Em virtude disso, uma mesma implementação que atenda a vários desses sistemas, padronizados ou não, é altamente desejável.

O presente trabalho tem por objetivo ilustrar os detalhes da implementação de um modem OFDM que possui uma melhor modularidade em hardware que aquela apresentada em [3]. Além disto, a presente arquitetura foi realizada de modo a permitir que os parâmetros do modem como quantidade de bits da sequência de dados, mapeamento QAM (Quadrature Amplitude Modulation) e número de pontos do modulador/demodulador IFFT (Inverse Fast Fourier Transform)/FFT (Fast Fourier Transform) possam ser facilmente modificados conforme configuração desejada.

\section{TÉCNICA OFDM}

A técnica multiportadora OFDM divide a seqüencia de dados a ser transmitida em várias subseqüências que, paralelamente moduladas em igual número de portadoras, são transmitidas simultaneamente em subcanais idealmente ortogonais. $\mathrm{O}$ fato da modulação e demodulação serem realizadas

Euripedes Rocha e Jair A. L. Silva, Laboratório de Telecomunicações, Departamento de Engenharia Elétrica, Universidade Federal do Espírito Santo, Vitória, Brasil, E-mails: arquiravnos@gmail.com, djaiscv@ hotmail.com. pelos algoritmos rápidos IFFT/FFT reduz a complexidade computacional de um transceptor, tornando possível a sua implementação prática.

\section{A. Implementação}

A implementação do modem OFDM foi feita em linguagem VHDL (VHSIC Hardware Description Language) e possui a arquitetura ilustrada na Figura 1.

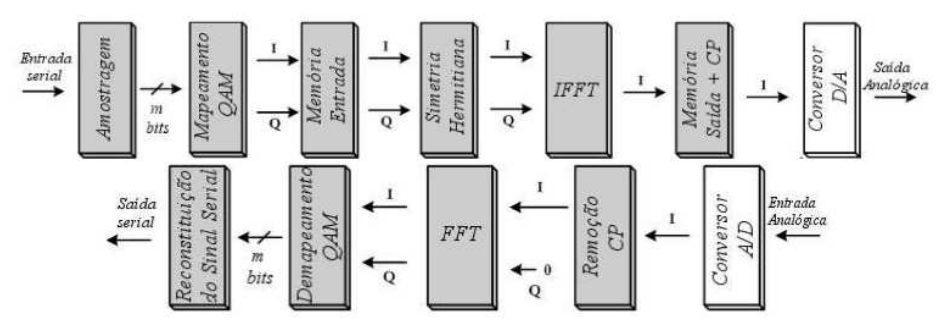

Fig. 1. Arquitetura de hardware utilizada para implementação do modem OFDM. A configuração ilustra o teste back to back realizado.

A modulação por subportadora ou o mapeamento da seqüência de bits de informação escolhido foi o 4-QAM (Quadrature Amplitude Modulation) que pode ser facilmente ampliado dada a modularidade do transmissor implementado. Na etapa posterior há um buffer que garante a entrada contínua de dados. Segue-se a implementação da simetria Hermitiana, a qual garante coeficientes reais na saída do modulador realizado pelo sub-sistema digital IFFT. O receptor realiza o processo inverso não possuindo, entretanto, um estágio que desfaça a simetria Hermitiana uma vez que esta se desfaz pelas próprias propriedades da transformada de Fourier [3], [4]. Portanto, existe um bloco que realiza a FFT dos coeficientes reais da saída do transmissor, e um que efetua o demapeamento QAM dos subsímbolos complexos e simétricos provenientes da FFT. Até o presente momento, o receptor foi testado apenas na forma back-to-back, ou seja nenhum canal foi utilizado na transmissão. Ressalta-se que o processamento envolvido na implementação da IFFT/FFT foi atendido pelo Radix-4 [5]. Destaca-se ainda a generalidade do código, pois simples mudanças de configuração de parâmetros permitem utilizar diferentes números de pontos na IFFT/FFT e por conseqüência gerar diferentes símbolos OFDM.

\section{B. Ocupação}

Ao sintetizar um código para um FPGA específico é possível verificar como foi realizada a ocupação do hardware em questão. O hardware utilizado foi o FPGA Virtex-2, xc2v3000 , da Xilinx com o kit Xtreme DSP da Agilent. Este FPGA possui 3 Mega portas lógicas, e 96 blocos de 18 
Kbits de RAM. O relatório de ocupação permite analisar a capacidade do hardware e a freqüência máxima de relógio. A Tabela I mostra o relatório de ocupação para diversos números de pontos da IFFT, na implementação do transmissor.

TABELA I

RELATÓRIO DA OCUPAÇÃO PELO NÚMERO DE PONTOS DA IFFT.

\begin{tabular}{|c||c|c|c|c|c|}
\hline & 64 & 256 & 1024 & 4096 & Total \\
\hline \hline Slices & $4.10 \%$ & $4.97 \%$ & $5.82 \%$ & $6.47 \%$ & 14336 \\
\hline Flip-Flops & $2.99 \%$ & $3.54 \%$ & $4.06 \%$ & $4.58 \%$ & 28672 \\
\hline 4 Inout LUT & $3.79 \%$ & $4.60 \%$ & $5.44 \%$ & $6.07 \%$ & 28672 \\
\hline Bounded IOB & $4.13 \%$ & $4.13 \%$ & $4.13 \%$ & $4.13 \%$ & 484 \\
\hline Block RAM & $5.21 \%$ & $5.21 \%$ & $5.21 \%$ & $16.67 \%$ & 96 \\
\hline GCLK & $6.25 \%$ & $6.25 \%$ & $6.25 \%$ & $6.25 \%$ & 16 \\
\hline
\end{tabular}

A Tabela II ilustra a análise de ocupação de hardware do modem completo, transmissor e receptor.

TABELA II

RELATÓRIO DA OCUPAÇÃo PELO NÚMERO DE PONTOS DA IFFT/FFT.

\begin{tabular}{|c||c|c|c|c|c|}
\hline & 64 & 256 & 1024 & 4096 & Total \\
\hline \hline Slices & $8.10 \%$ & $9.64 \%$ & $11.37 \%$ & $12.65 \%$ & 14336 \\
\hline Flip-Flops & $6.15 \%$ & $7.18 \%$ & $8.29 \%$ & $9.39 \%$ & 16384 \\
\hline 4 Inout LUT & $7.38 \%$ & $9.02 \%$ & $10.68 \%$ & $11.91 \%$ & 28672 \\
\hline Bounded IOB & $0.62 \%$ & $0.62 \%$ & $0.62 \%$ & $0.62 \%$ & 484 \\
\hline Block RAM & $8.33 \%$ & $8.33 \%$ & $8.33 \%$ & $26.04 \%$ & 96 \\
\hline GCLK & $6.25 \%$ & $6.25 \%$ & $6.25 \%$ & $6.25 \%$ & 16 \\
\hline
\end{tabular}

Alterando o número de pontos do conjunto IFFT e FFT é possível observar que devido à sua arquitetura e à síntese do FPGA, não existe muita variação na ocupação. É possível perceber também que a ocupação se torna crítica pela quantidade de memórias RAM pois, quando passou de 1024 para 4096 portadoras exigiu-se a alocação de mais blocos de memória RAM. Outra observação importante, com relação à eficiência do código, é a taxa de clock máxima permitida pelo hardware, pois, para um aumento de 64 vezes no número de pontos, o clock máximo permitido pelo hardware decresceu apenas $13,11 \%$ (de $180.23 \mathrm{MHz}$ em 64 pontos para $156.61 \mathrm{MHz}$ em 4096 pontos).

\section{Resultados}

Com o objetivo de averiguar o resultado do modem implementado, além do teste back to back, um sinal OFDM na saída do transmissor foi medido por um osciloscópio digital. Parametrizado em 31 subsímbolos gerados por uma constelação 4-QAM e provenientes de uma seqüencia de 64 bits, e utilizando um modulador de 64 pontos gerou-se o símbolo da Figura 2.

Com duração útil do símbolo $T_{u} \cong 914 n s$, largura de banda $B W \cong 35 \mathrm{MHz}$ e subportadoras espaçadas em $\Delta_{f}=\frac{1}{T_{u}}=$ $\frac{2 B W}{32} \cong 1.1 \mathrm{MHz}$, o sinal da Figura 2 ilustra a natureza de a excursão e o espectro de um símbolo de um sistema multiportadora OFDM com taxa de transferência $R_{s}=\frac{64}{T_{u}} \cong$ $70 \mathrm{Mbps}$ [6], [7]. Vale ressaltar que na saída do FPGA existe um conversor D/A de faixa de amplitude compreendida entre -2 e 2 Volts.
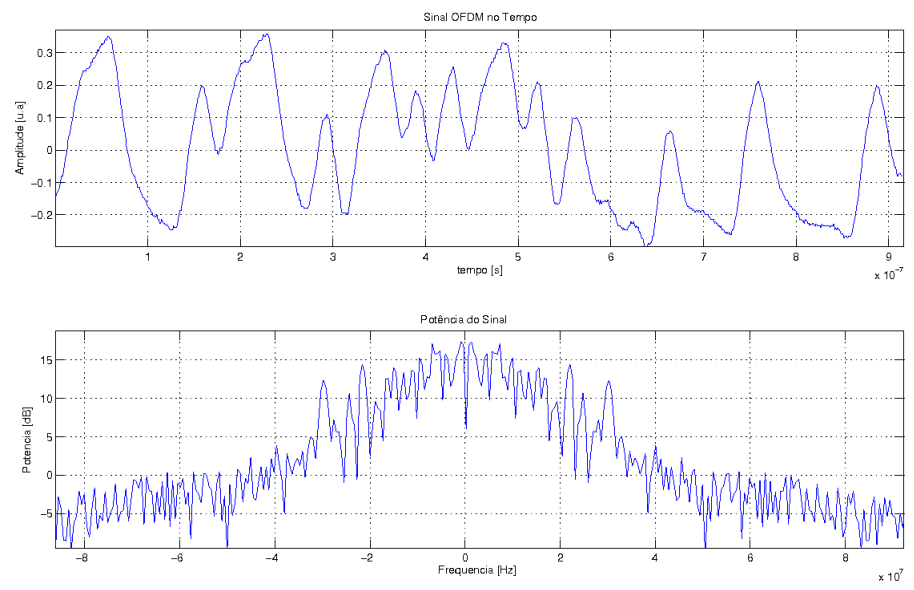

Fig. 2. Sinal OFDM gerado na saída do transmissor.

\section{CONCLUSÕES}

A idéia de implementação de um modem OFDM totalmente digital é perfeitamente viável e prática uma vez que, utilizando-se uma FPGA como elemento final de implementação, os testes e futuras melhorias tornam-se simples. A arquitetura modular implementada possibilitou um enorme avanço nas atualizações de cada função e até da capacidade do sistema. No que tange à ocupação em hardware ficou evidente que o aumento no número de pontos não é crítico, pois basicamente, só a quantidade de memória RAM é afetada.

\section{REFERÊNCIAS}

[1] S. Hara, R. Prasad Multicarrier Techniques for 4G Mobile Communications. Artech House Inc, Norwood, MA, USA, 2003.

[2] L. Hanzo, L. Webb, W. Keller Single- and Multi-carrier Quadrature Amplitude Modulation : Principles and Applications for Personal Communications, WLANs and Broadcasting. John Wiley and Sons, Baffins Lane, England, 2000.

[3] Siqueira, T. M.Implementação de um MODEM OFDM em FPGA. Projeto de Graduação, Departamento de Engenharia Elétrica, Universidade Federal do Espírito Santo, 2004.

[4] Silva, J. A. L. Análise de desempenho de um sistema COFDM para comunicação via rede elétrica.. Dissertação de Mestrado, Programa de Pós-Graduação em Engenharia Elétrica, Universidade Federal do Espírito Santo, 2006.

[5] Ming, Z. Radix 4 Complex FFT. Disponível em Julho de 2006 no site www.opencores.org.

[6] J. A. L. Silva, T. M. Siqueira, M. R. N. Ribeiro, R. V. Andreão, E. O. T. Salles, M. E. V. Segatto, A Comparative Analysis of the Performance of OFDM and COFDM Indoor PLC Systems. ICT $2006-13^{\text {th }}$ Conference on Telecommunications, Funchal, v. 1, 2006.

[7] T. L. Dezan, J. A. L. Silva, K. F. Coco, T. M. Siqueira, E. O. T. Salles, M. E. V. Segatto, Modulação OFDM Aplicada a Power Line Communication. XXI Simpósio Brasileiro de Telecomunicações, Belém/Pa. v. 1. p. 1-6, 2004. 